\title{
Ängste und Depression bei Adoleszenten mit juveniler idiopathischer Arthritis
}

\author{
Hanns L et al. Association of Anxiety With Pain \\ and Disability but Not With Increased Measures \\ of Inflammation in Adolescent Patients With \\ Juvenile Idiopathic Arthritis. Arthritis Care Res \\ (Hoboken) 2020; 72: 1266-1274. doi: 10.1002/ \\ acr.24006
}

Die Adoleszenz stellt im Hinblick auf psychiatrische Erkrankungen eine vulnerable Lebensphase dar. Gilt dies auch für Patienten mit einer juvenilen idiopathischen Arthritis (JIA)? Besteht ein Zusammenhang zwischen Ängsten und Depressionen und den klinischen JIAManifestationen? Und inwiefern wirken sich die psychischen Belastungen auf Entzündungsmediatoren aus? Diesen und anderen Fragen gingen britische Wissenschaftler nach.

Sie befragten 136 JIA-Patienten im Alter zwischen 13 und 18 Jahren im Rahmen ihrer ambulanten rheumatologischen Betreuung am University College London mithilfe des „Mood and Feelings Questionnnaire“ sowie 
mithilfe des „State-Trait Anxiety Inventory“ zu depressiven Symptomen und Angstbelastungen. Keiner der Probanden war kürzlich mit Steroiden behandelt worden. Von allen JIA-Patienten lagen Informationen zur Krankheitsaktivität, zum Allgemeinzustand, zu Leistungseinschränkungen, zur Schmerzbelastung sowie zur Medikation vor. Ferner objektivierten die Forscher bei einem Teil der Patienten die Erythrozytensedimentationsrate, die CRP- und die Kortisol-Konzentration sowie die Konzentration proinflammatorischer Zytokine (Serum-Interleukin-6, Interleukin-6-Produktion nach Lipopolysaccharid-Stimulation peripherer mononukleärer Zellen). Das Vergleichskollektiv bildeten 88 gesunde Jugendliche gleichen Alters.

\section{Ergebnisse}

Die JIA-Patienten und die Kontrollen unterschieden sich im Hinblick auf den Grad der Angst- und Depressionsbelastung nicht wesentlich. In der Gruppe der JIA-Patienten zeigte sich ein signifikanter Zusammenhang zwischen der Angstsymptomatik und Behinderungen, Schmerzen sowie dem Ergebnis des „Physician Global Assessment“ (visuelle Analogskala), nicht jedoch mit der Anzahl aktiver Gelenke. Depressive Symptome korrelierten ebenfalls mit Behinderungen und Schmerzen, bezüglich des Ergebnis des „Physician Global Assessment“ bestand hier allerdings nur eine tendenzielle Korrelation. Die Auswertung der Laborproben ergab: Im Kollektiv der JIA-Patienten bestand kein Zusammenhang zwischen der Angstbelastung und irgend einem der untersuchten Entzündungsmarker. Gleiches galt für die depressive Symptomatik. Im Kollektiv der gesunden Kontrollen deutete sich eine Korrelation zwischen der Angstbelastung und der stimulierten Interleukin-6-Produktion an, statistische Signifikanz bestand hier allerdings nicht.

\section{FAZIT}

Jugendliche JIA-Patienten erleben ähnlich starke Ängste und Depressionen wie gesunde Adoleszente, so die Autoren. Beide psychischen Belastungen scheinen bei einer JIA mit den Schmerzen, den Einschränkungen sowie dem Allgemeinzustand, nicht jedoch mit der Entzündungsaktivität, zu korrelieren. Die Betroffenen, so ihre Einschätzung, profitieren von der Betreuung durch pädiatrisch-rheumatologische Psychologen, wobei eine enge Zusammenarbeit mit Physiotherapeuten und Arbeitsmedizinern wünschenswert ist.

Dr. med. Judith Lorenz, Künzell 